\title{
Medical Disclaimer
}

This book concerns the natural and cultural history of Texas plants; it is not meant to be a guide to plant uses. This is especially true for plants with reported medical or psychotropic properties. Such properties are here reported for their historical value, to enlighten the public about the treatments of an earlier time and, in some cases, about their legacies today. In no way is this book meant to be prescrip- tive, and it by no means replaces professional medical advice. The medical uses contained herein should not be read as promoting experimental use by individuals, who could do serious harm to themselves. Neither the author nor the University of Texas Press accepts responsibility for the accuracy of the information itself, or for the consequences from the use or misuse of the information in this book. 